\title{
Regular Wave Propagation Out of Noise in Chemical Active Media
}

\author{
S. Alonso, ${ }^{1,2, *}$ I. Sendiña-Nadal, ${ }^{3}$ V. Pérez-Muñuzuri, ${ }^{3}$ J. M. Sancho, ${ }^{2}$ and F. Sagués ${ }^{1}$ \\ ${ }^{1}$ Departament de Química Física, Universitat de Barcelona, Avenida Diagonal 647, 08028 Barcelona, Spain \\ ${ }^{2}$ Departament d'Estructura i Constituents de la Matèria, Universitat de Barcelona, Avenida Diagonal 647, 08028 Barcelona, Spain \\ ${ }^{3}$ Grupo de Física non Lineal, Facultade de Física, Universidade de Santiago de Compostela, 15706 Santiago de Compostela, Spain \\ (Received 1 February 2001; published 30 July 2001)
}

\begin{abstract}
A pacemaker, regularly emitting chemical waves, is created out of noise when an excitable photosensitive Belousov-Zhabotinsky medium, strictly unable to autonomously initiate autowaves, is forced with a spatiotemporal patterned random illumination. These experimental observations are also reproduced numerically by using a set of reaction-diffusion equations for an activator-inhibitor model, and further analytically interpreted in terms of genuine coupling effects arising from parametric fluctuations. Within the same framework we also address situations of noise-sustained propagation in subexcitable media.
\end{abstract}

DOI: 10.1103/PhysRevLett.87.078302

PACS numbers: $82.40 . \mathrm{Bj}, 05.40 . \mathrm{Ca}, 47.54 .+\mathrm{r}$

Since their discovery thirty years ago [1], target patterns have constituted one of the most distinctive and visually compelling examples of self-organization in chemical systems. Somewhat more general, control on wave initiation and propagation may have a wealth of potential implications not only for chemical [2-4] or biochemical systems [5,6], but extending to cardiology [7] or neurophysiology [8] contexts. Although unavoidably present in any realistic situation of these scenarios, the minimization of noise and disorder is always pursued under the rationale that their effects may, if not destroy, at least largely mask, the intrinsic spatiotemporal regularities of any such wave propagation phenomena. Here, contrarily, we provide ex- perimental and numerical evidence, and also an analytical explanation, of just the opposite, now beneficial, influence, by showing that an excitable chemical system may rectify external fluctuations into regularly organized wave trains. Explicitly, we will show that the photosensitive BelousovZhabotinsky (BZ) reaction [9], under excitable conditions unable to create autowaves, does maintain a target structure when subjected to a patterned and continuously evolving random illumination.

Moreover a theoretical framework for activatorinhibitor models will be proposed to interpret not only this experimental finding but the recent related one of noisesupported waves in subexcitable media [10]. With this (a)

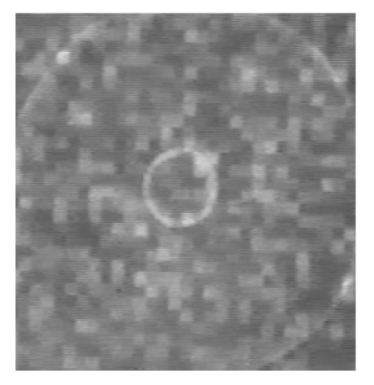

(a')

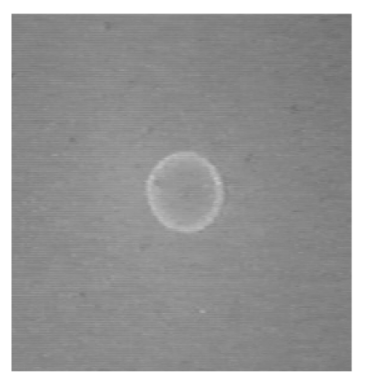

(b)

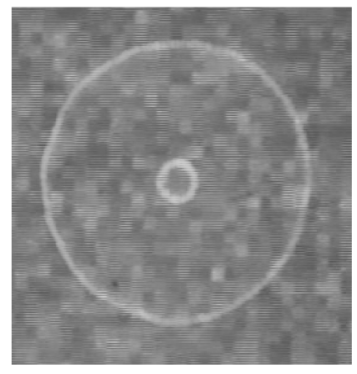

(b')

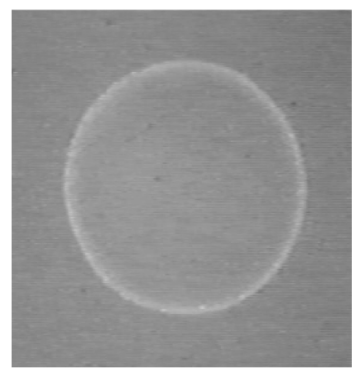

(c)

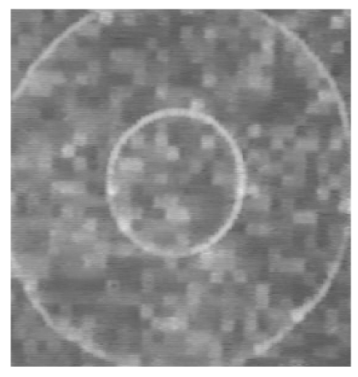

(c')

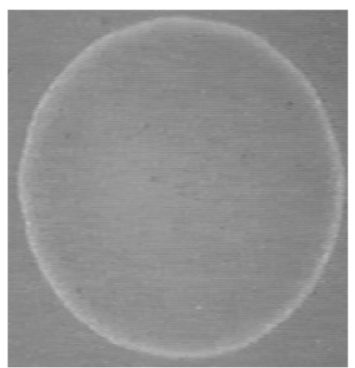

FIG. 1. Sequence of images, (a)-(c), showing a noise-sustained target pattern in the BZ reaction. The medium is forced with a spatiotemporal fluctuating illumination. The average light intensity corresponds to excitable conditions $\left(I_{0}=0.29 \mathrm{~mW} / \mathrm{cm}^{2}\right)$. By comparison, images $\left(\mathrm{a}^{\prime}\right)-\left(\mathrm{c}^{\prime}\right)$ correspond to a fluctuation-free case with a constant and uniform illumination fixed to the averaged intensity $I_{0}$. Images shown in the figure correspond to an exposed area of $1.38 \times 1.36 \mathrm{~cm}^{2}$. Images $(\mathrm{a}) /(\mathrm{b})\left[\left(\mathrm{a}^{\prime}\right) /\left(\mathrm{b}^{\prime}\right)\right]$ are separated by $40 \mathrm{~s}$ and $(\mathrm{b}) /(\mathrm{c})\left[\left(\mathrm{b}^{\prime}\right) /\left(\mathrm{c}^{\prime}\right)\right]$ by $20 \mathrm{~s}$. 
unified perspective we highlight the specificities of these phenomenologies in relation to other celebrated noise-constructive effects, typical of either forced or autonomous extended systems, i.e., array enhanced stochastic resonance [11,12], taming spatiotemporal chaos with disorder [13], noise-enhanced signal propagation $[14,15]$, and noise-induced synchronization to global oscillations for arrays of excitable units $[16,17]$ or coherence resonance oscillators $[18,19]$.

Experiments were carried out using thin $(0.3 \mathrm{~mm})$, squared $\left(3 \times 3 \mathrm{~cm}^{2}\right)$ film of silica gel, in which the light-sensitive catalyst, ruthenium-bipyridyl $[R u(b p y)]$, was immobilized [9] [solution of $15 \%$ sodium silicate, $1.5 \mathrm{mMRu}(\mathrm{bpy})_{3}^{2+}$ and $\left.1 M \mathrm{H}_{2} \mathrm{SO}_{4}\right]$ prepared as in Ref. [20]. A solution of catalyst-free BZ reaction was poured onto the gel (initial concentrations: $0.15 \mathrm{M} \mathrm{KBr}$, $0.34 M$ malonic acid, $0.38 M \mathrm{NaBrO}_{3}$, and $0.48 M \mathrm{H}_{2} \mathrm{SO}_{4}$ ). The temperature was kept constant at $25 \pm 1{ }^{\circ} \mathrm{C}$. The light intensity reaching the gel governs the system excitability through the production of $\mathrm{Br}^{-}$[21,22]. Images of the patterns, whose contrast was enhanced with an interference filter of $460 \mathrm{~nm}$, were captured in a video recorder through a CCD camera placed vertically.

Spatiotemporal structured noise is introduced into the system by using a computer controlled and continuously evolving fluctuating patterned illumination, which is video projected (from below) onto the gel film [23]. The pattern of illumination consisted of an array of square cells of linear size $l(l=0.44 \mathrm{~mm})$, each of them obeying a Gaussian statistics $\left(\sigma=0.14 \mathrm{~mW} / \mathrm{cm}^{2}\right)$ around a prescribed reference illumination value $I_{0}$ for excitable conditions $\left(I_{0}=0.29 \mathrm{~mW} / \mathrm{cm}^{2}\right)$. The different illumination values are reproduced through an eight-bit gray scale between 0 and 255 that is filtered by means of a video projector according to a calibration curve. All the cells are independently updated according to the smallest time provided by the computer (100 ms), to reproduce fluctuations with extremely small correlation time (mimicking an effective white noise limit). To assure a radial distribution of the refractory time, a central black spot $\left(I_{0}=0\right.$, $R \approx 1.5 \mathrm{~mm}$ ) (whose size was found to be critical) was projected into the sample until a circular wave emerged from this local perturbation. Structured light fluctuations were subsequently imposed all over the system, and when prescribed above an intensity threshold, repeated wave nucleation, leading to a circular wave train, was observed, as shown in Fig. 1. For the sake of comparison, we include in the same figure the case of uniform and steady illumination at the same reference value. As expected, a single autowave propagating from the initial condition is found in the absence of fluctuations.

Actually, repeated wave emission could be sustained only for an intermediate range of fluctuation intensities. For low noise level, no periodic wave emission was observed. Contrarily, under a too high noisy environment, sustained wave initiation was possible but immediately waves were continuously broken into scattered fragments progressively occupying the whole medium. Quantitative results, summarized in Fig. 2, reproduce a monotonous decrease of the emission period as the noise level increases. Contrarily, there exists a critical noise intensity approaching which the emission period diverges. For lower intensity fluctuations the system will stay forever under purely excitable conditions. Regarding the role of the cell size, the best results were observed when comparable to the wave width. With half this value, which corresponds to the size of an illumination pixel, waves hardly nucleated, whereas for larger sizes no waves were generated, probably due to the progressive loss of the patterned structure of the noise.

Numerical studies of noise-sustained targetlike patterns were conducted using the photosensitive versions [24] of the complete three-variable Oregonator model [25], as well as its reduced form [26] that follows from the adiabatic elimination of the rapidly varying $\mathrm{Br}^{-}$concentration. Although both reproduce the experimental observations, on what follows we limit ourselves to this latter level of
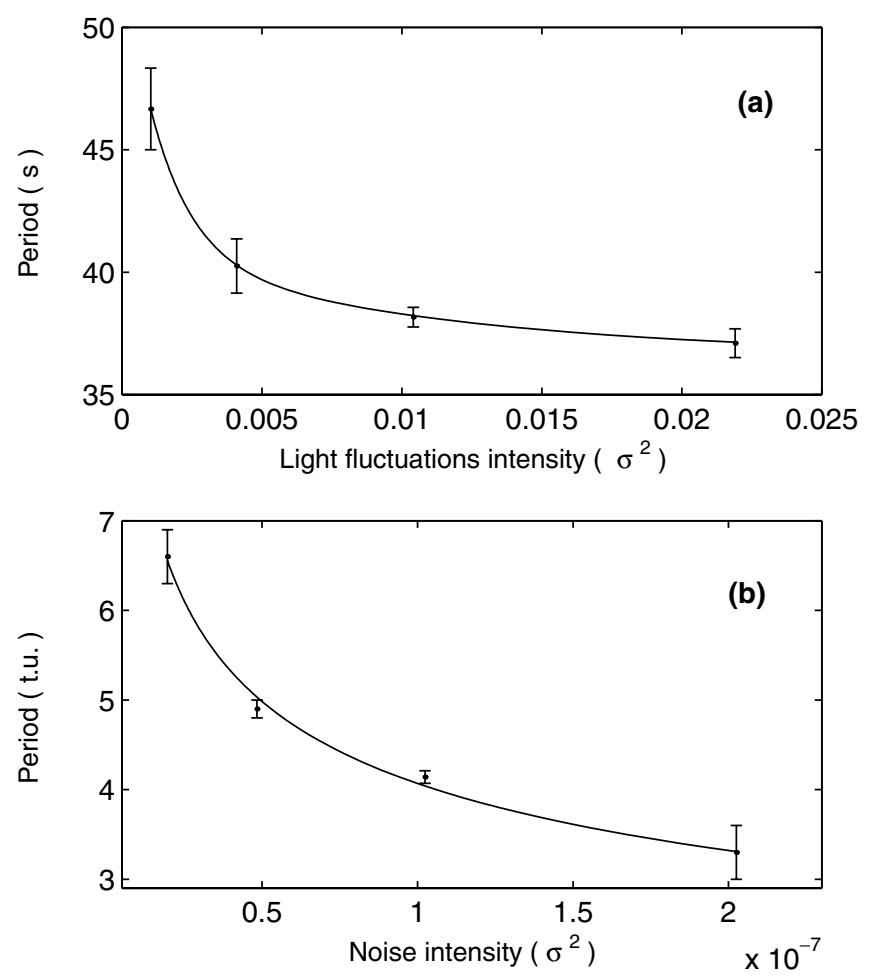

FIG. 2. Dependence of the wave nucleation period on the noise intensity: (a) experimental results, (b) numerical results obtained from the two-variable dimensionless Oregonator model. Continuous lines are simply to guide the eye. In the experimental case periods are determined by averaging the time intervals between consecutive waves of the $\mathrm{BZ}$ reaction. In the numerical situation, periods are computed from successive circular waves passing through four square symmetrically distributed points separated 150 pixels from the center position. An average is taken over two realizations of the initial condition. The model equations were integrated in a two-dimensional rectangular grid of $320 \times 320$ pixels (unit grid size $\Delta=0.2$ ) with no-flux boundary conditions. The set of parameters were $\epsilon=0.03, q=0.0015, f=1$, and $\delta=1$. 
modelization since the theoretical considerations proposed below may widen their scope when applied to activatorinhibitor schemes and even admit in this case a fully analytic formulation. The model equations thus read

$$
\begin{aligned}
& \partial_{t} u=\delta \nabla^{2} u+\frac{1}{\epsilon}\left(u-u^{2}-(f v+\phi) \frac{u-q}{u+q}\right), \\
& \partial_{t} v=u-v,
\end{aligned}
$$

where the variables $u$ and $v$ stand, respectively, for the dimensionless concentrations of $\mathrm{HBrO}_{2}$ and $\mathrm{Ru}(\mathrm{bpy})_{3}^{2+} . \delta$ is the diffusion coefficient for $u, \epsilon$ and $q$ are parameters related to the kinetics of the reaction, $f$ is an adjustable stoichiometric factor, and $\phi$ is the parameter proportional to the illumination intensity. Corresponding to the experiments above, the fluctuating excitability was introduced through a random distribution of the local values of $\phi$. The pattern consisted of an array of square cells of $1 \times 1$ pixels ( 0.2 dimensionless space units), each of them obeying a Gaussian statistics around a fixed reference value $\phi_{0}$ in the excitable range $\left(\phi_{0}=0.001\right)$. All the cells were independently updated every time step (0.0005 dimensionless time units) to simulate uncorrelated fluctuations (white noise limit) [27]. Using initial preparations similar to those in the experiments, conditions to observe periodic wave emission were also found for an intermediate range of fluctuation intensities. Actually, a series of simulations carried out to determine the dependence of the observed periodicity of the emitted waves on noise intensity is also reproduced in Fig. 2, showing similar trends to those observed in the experiments. A particular realization of such numerical simulations is displayed in panel (a) of Fig. 3, as compared with the noise-free case for the same $\phi_{0}$ value.

The keystone to theoretically interpret the phenomenon just reported consists in realizing that when noise is introduced through the illumination parameter in the model equations, a random term left from the fluctuating zero mean part enters multiplicatively into the dynamics of the activator variable (in our case the $\mathrm{HBrO}_{2}$ ) [28]. However, due to this nonlinear coupling, a systematic genuine nonzero contribution arises when averaging such a noisy

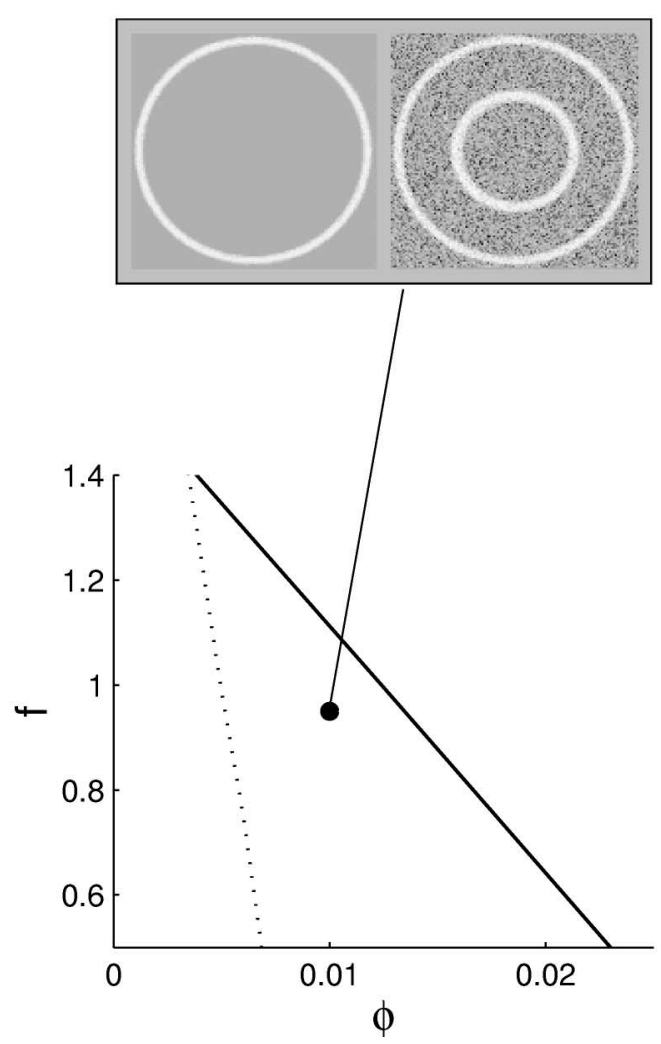

(a)

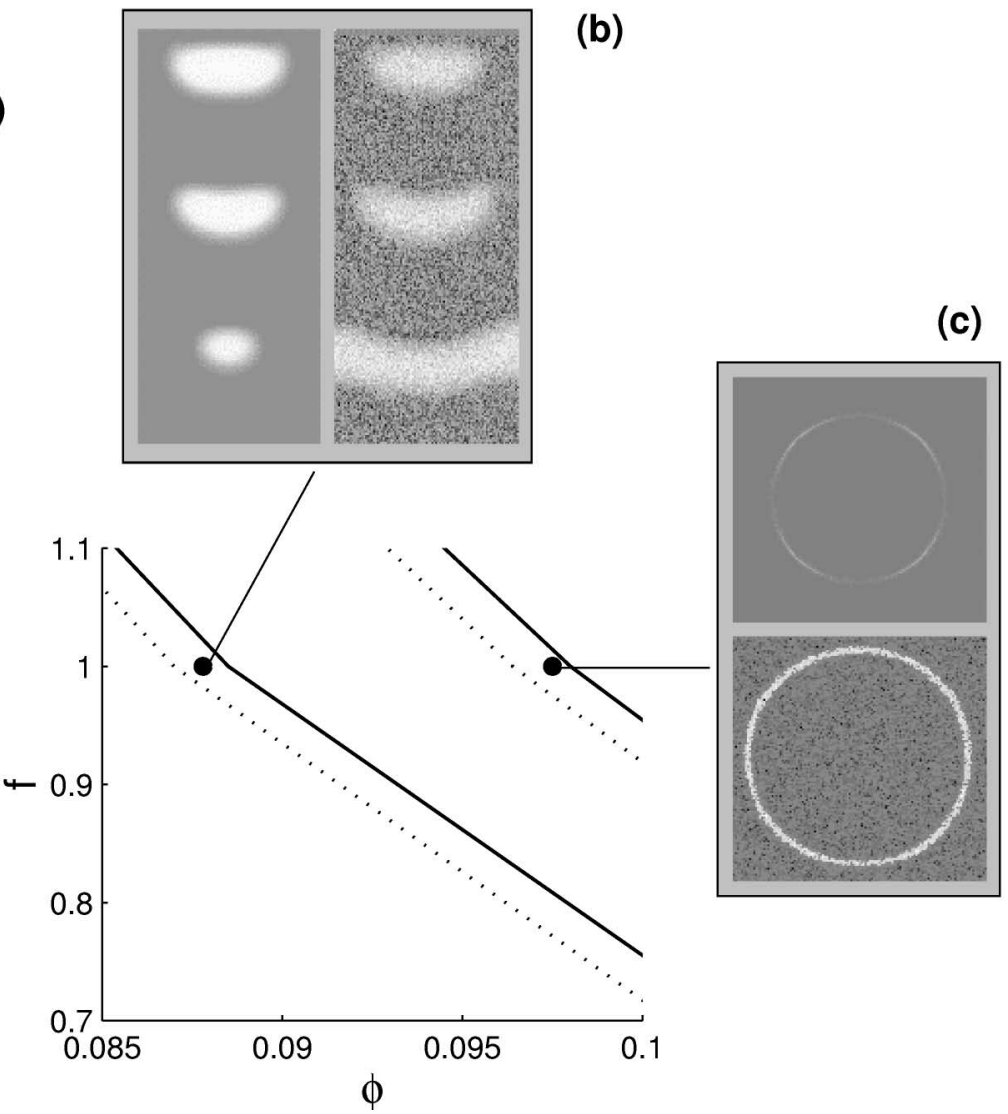

FIG. 3. Diagram classifying the different dynamical regimes of the two-variable Oregonator model (dashed lines) Eq. (1) and the effective two-variable model (solid lines) Eq. (2). In the graph on the left the boundaries between oscillatory and excitable regimes are shown. In the graph on the right the boundaries among excitable, subexcitable (shrinking of 2D wave segments), and nonexcitable (collapse of 1D waves) conditions are shown. Panels accompanying the diagram are representative of the three noise-mediated transitions referred to in the paper for a particular value of $\sigma^{2}=1.4 \times 10^{-4}$. They represent three different couples of noise-free/noise-forced systems, each panel with the same averaged value of the illumination parameter $\left(\phi_{\text {excitable/osc. }}=0.005\right.$, $\phi_{\text {subexcitable/exc. }}=0.087, \phi_{\text {nonexcitable/subexc. }}=0.096$ ). Note that panel (b), at variance with (a) and (c), is built up from a superposition of snapshots. Concentrations of $u$ are represented by gray levels, higher/smaller concentration values corresponding to lighter/darker color pixels. 
term in relation to the dynamics of the activator variable. Given the finite, although small, time and length scales of the experimental fluctuations and for moderate noise intensities, one can incorporate the dominant noise contributions which correspond to the lowest order term of an expansion on these parameters as a correction to those kinetic terms, discarding additional nonsystematic fluctuating terms which average to zero [29]. As a result we finally end up with an effective totally deterministic two-variable Oregonator model whose explicit equations read

$$
\begin{aligned}
\partial_{t} u= & \delta \nabla^{2} u+\frac{1}{\epsilon}\left(u-u^{2}-\left(f v+\phi_{0}\right) \frac{u-q}{u+q}\right) \\
& +\frac{1}{\epsilon}\left(\frac{\sigma^{2}}{\Delta^{2}} \frac{2 q}{\epsilon(u+q)^{2}} \frac{u-q}{u+q}\right), \\
\partial_{t} v= & u-v,
\end{aligned}
$$

in terms of the noise intensity $\sigma^{2}$ and the pixel size $\Delta$. Once in this form, the excitable and oscillatory properties of such a renormalized model are easily computed. This whole procedure enables us, as shown in Fig. 3, to predict a noise intensity dependent shift of the boundaries separating the dynamical regimes (oscillatory, excitable, subexcitable, and nonexcitable) of such an effective scheme as compared to the noise-free Oregonator model. Notice that, in fact, such a theoretical framework enables us to interpret not only the appearance of noise-induced target patterns, but related scenarios of noise-supported wave propagation, both in subexcitable [panel (b)] [10] and nonexcitable conditions [panel (c)] of Fig. 3 [30]. In other words an excitable (respectively, subexcitable or nonexcitable) propagating condition in a noise-free environment turns into an oscillatory (respectively, excitable or subexcitable) situation when external uncorrelated spatiotemporal fluctuations of zero mean and appropriate intensities are superimposed on the illumination parameter.

As a final comment, note that the whole argument just presented highlights the delicate coupling between noise and the nonlinearities of the chemical system, which, rather than resulting into new noise-induced periodicities, evoke its intrinsic "eigenvalues," both temporal (emission frequency) and spatial (period of the wave train). In this respect, this theoretical interpretation is essentially different from those based on barrier crossing dynamics arguments that are commonly invoked to explain the statistical periodicities observed in related stochastic resonance [11] or coherence resonance [19] phenomena in spatially extended systems.

We wish to thank J. García-Ojalvo for fruitful discussions. This work was supported by the Comisión Interministerial de Ciencia y Tecnología (DGES) and Comissionat per a Universitat i Recerca (Generalitat de Catalunya) under Projects No. BXX2000-0638, No. BFM2000-0624, No. PB97-0540, No. BFM20000348, and No. 1999SGR00041.
*Corresponding author.

Email address: s.alonso@qf.ub.es

[1] A. N. Zaikin and A. M. Zhabotinskii, Nature (London) 225, 535 (1970).

[2] J. Ross, S.C. Müller, and C. Vidal, Science 240, 460 (1988).

[3] A. T. Winfree, Science 175, 634 (1972).

[4] R. Kapral and K. Showalter, Chemical Waves and Patterns (Kluwer, Dordrecht, 1995).

[5] Physica (Amsterdam) 49D, No. 1/2 (1991), edited by H. L. Swinney and V. I. Krinsky.

[6] A. Goldbeter, Biochemical Oscillations and Cellular Rhythms (Cambridge University Press, Cambridge, U.K., 1996).

[7] A. V. Panfilov and A. V. Holden, Computational Biology of the Heart (John Wiley \& Sons, Chichester, 1997).

[8] P. Jung, A. H. Cornell-Bell, K. Madden, and F. Moss, J. Neurophys. 79, 1098 (1998).

[9] L. Kuhnert, K. J. Agladze, and V. I. Krinsky, Nature (London) 337, 244 (1989).

[10] S. Kádár, J. Wang, and K. Showalter, Nature (London) 391, 770 (1998).

[11] J. F. Lindner, B. K. Meadows, W. L. Ditto, M. E. Inchiosa, and A. R. Bulsara, Phys. Rev. Lett. 75, 3 (1995).

[12] M. Löcher, G. A. Johnson, and E. R. Hunt, Phys. Rev. Lett. 77, 4698 (1996).

[13] Y. Braiman, J. F. Lindner, and W. L. Ditto, Nature (London) 378, 465 (1995).

[14] M. Löcher, D. Cigna, and E. R. Hunt, Phys. Rev. Lett. 80, 5212 (1998).

[15] J. F. Lindner, S. Chandramouli, A. R. Bulsara, M. Löcher, and W. L. Ditto, Phys. Rev. Lett. 81, 5048 (1998).

[16] C. Kurrer and K. Shulten, Phys. Rev. E 51, 6213 (1995).

[17] H. Hempel, L. Schimansky-Geier, and J. García-Ojalvo, Phys. Rev. Lett. 82, 3713 (1999).

[18] S. K. Han, T. G. Yim, D. E. Pastrov, and O. V. Sosnovtseva, Phys. Rev. Lett. 83, 1771 (1999).

[19] A. Neiman, L. Schimansky-Geier, A. Cornell-Bell, and F. Moss, Phys. Rev. Lett. 83, 4896 (1999).

[20] T. Yamaguchi, L. Kuhnert, ZS. Nagy-Ungvaray, S. C. Müller, and B. Hess, J. Phys. Chem. 95, 5831 (1991).

[21] S. Kádár, T. Amemiya, and K. Showalter, J. Phys. Chem. A 101, 8200 (1997).

[22] V. K. Vanag, A. M. Zhabotinskii, and I. R. Epstein, J. Phys. Chem. A 104, 8207 (2000).

[23] I. Sendiña-Nadal et al., Phys. Rev. Lett. 84, 2734 (2000).

[24] H. J. Krug, L. Pohlmann, and L. Kuhnert, J. Phys. Chem. 94, 4862 (1990).

[25] R. J. Field and R. M. Noyes, J. Chem. Phys. 60, 1877 (1974).

[26] J. J. Tyson and P. C. Fife, J. Chem. Phys. 73, 2224 (1980).

[27] J. García-Ojalvo and J.M. Sancho, Noise in Spatially Extended Systems (Springer-Verlag, New York, 1999).

[28] A. S. Mikhailov, Phys. Rep. 184, 307 (1989).

[29] M. A. Santos and J. M. Sancho (to be published).

[30] More experimental images are available at http://fmmeteo.usc.es/ irene/web/noisetargets.htm 